University of Nebraska - Lincoln

DigitalCommons@University of Nebraska - Lincoln

Faculty Publications, Department of Physics and Astronomy

10-26-2004

\title{
Surface structure of ultrathin copolymer films of ferroelectric vinylidene fluoride $(70 \%)$ with trifluoroethylene $(30 \%)$ on graphite
}

\author{
Lei Cai \\ Florida International University, Icai@broward.org \\ Hongwei Qu \\ University of Florida, qu2@oakland.edu \\ Chenxi Lu \\ Florida International University, Chenxi.Lu@fiu.edu \\ Stephen Ducharme \\ University of Nebraska-Lincoln, sducharme1@unl.edu \\ Peter A. Dowben \\ University of Nebraska-Lincoln, pdowben@unl.edu \\ See next page for additional authors
}

Follow this and additional works at: https://digitalcommons.unl.edu/physicsfacpub

Part of the Physics Commons

Cai, Lei; Qu, Hongwei; Lu, Chenxi; Ducharme, Stephen; Dowben, Peter A.; and Zhang, Jiandi, "Surface structure of ultrathin copolymer films of ferroelectric vinylidene fluoride (70\%) with trifluoroethylene (30\%) on graphite" (2004). Faculty Publications, Department of Physics and Astronomy. 12.

https://digitalcommons.unl.edu/physicsfacpub/12

This Article is brought to you for free and open access by the Research Papers in Physics and Astronomy at DigitalCommons@University of Nebraska - Lincoln. It has been accepted for inclusion in Faculty Publications, Department of Physics and Astronomy by an authorized administrator of DigitalCommons@University of Nebraska Lincoln. 


\section{Authors}

Lei Cai, Hongwei Qu, Chenxi Lu, Stephen Ducharme, Peter A. Dowben, and Jiandi Zhang 


\title{
Surface structure of ultrathin copolymer films of ferroelectric vinylidene fluoride (70\%) with trifluoroethylene $(\mathbf{3 0} \%)$ on graphite
}

\author{
Lei Cai,${ }^{1}$ Hongwei Qu,${ }^{1} *$ Chenxi Lu,${ }^{1}$ S. Ducharme, ${ }^{2}$ P. A. Dowben, ${ }^{2}$ and Jiandi Zhang ${ }^{1, \dagger}$ \\ ${ }^{1}$ Department of Physics, Florida International University, Miami, Florida 33199, USA \\ ${ }^{2}$ Department of Physics and Astronomy and Center for Materials Research and Analysis, University of Nebraska-Lincoln, Lincoln, \\ Nebraska 68588, USA
}

(Received 7 June 2004; published 26 October 2004)

\begin{abstract}
The structure and local structural distortions, through the polarization manipulation, of crystalline films of ferroelectric vinylidene fluoride $(70 \%)$ with trifluoroethylene $(30 \%)$ [P(VDF-TrFE)] copolymer on graphite were studied by scanning tunneling microscopy (STM). A quasispiral twist in $\mathrm{C}-\mathrm{C}$ bonds with rotations about the polymer chain axis was observed by high-resolution STM, indicating a surface relaxation of the strained copolymer films. Such a relaxation behavior appears to be linked to the observed local dipole rotations accompanied by the reversal of the local polarization with biasing the STM tip. A structure model is proposed based upon the observations.
\end{abstract}

DOI: $10.1103 /$ PhysRevB.70.155411

PACS number(s): 68.55.Jk, 77.84.Jd, 82.37.Gk, 77.22.Ej

\section{INTRODUCTION}

Manipulation of a single atom and molecule has been a compelling research goal in the field of molecular electronics for more than half of a century. Following the invention of scanning tunneling microscopy (STM) (Ref. 1) and atomic force microscopy (AFM), ${ }^{2}$ such a manipulation has been successfully undertaken for both inorganic atoms (molecules) $)^{3-6}$ and organic molecules. ${ }^{7-12}$ One interesting subject is the exploitation for reversible conductance transitions associated with a molecular reorientation induced by the tip of scanning probe microscope. ${ }^{7-12}$ For example, reorientation of individual molecules on a surface can generate bistable conductance switching ${ }^{7-12}$ that is of potential value in molecular electronic devices. ${ }^{13-15}$ To realize these technologies, it is essential to better understand the local structural, electronic, and optical properties at the atomic scale, in addition to the molecular dynamics.

One of the attractive materials for molecular electronics is the class of polymers or copolymers in which individual monomer or monomer clusters may be manipulated with a local field. The strong dipoles, presenting in ferroelectric polymer materials, serve as a nearly ideal playground for such field induced molecular manipulation. Polymer of Vinylidene fluoride $[\mathrm{P}(\mathrm{VDF})]$ and random copolymers of vinylidene fluoride with trifluoroethylene [P(VDF-TrFE)] consist of monomers with large dipole moment and exhibit strong ferroelectric properties, even in the ultrathin film limit, drawing much attention because of their application to electromechanical transducers and nonvolatile memories. ${ }^{16-34}$ In the ferroelectric state, below the first-order ferroelectric phase transition temperature $T_{C}{ }^{B}$ $=80{ }^{\circ} \mathrm{C},{ }^{17,18,23,33}$ the copolymer P(VDF-TrFE; 70\%:30\%) exhibits the properties typical of ferroelectricity, such as spontaneous electrical polarization, polarization reversal (switching), polarization hysteresis, etc. Furthermore, this first-order ferroelectric phase transition is almost independent of the film thickness in crystalline copolymer films, in- dicating the existence of two-dimensional ferroelectricity. ${ }^{24,26,35-37}$ A first-order surface ferroelectric phase transition was also observed at $T_{C}{ }^{S}=20^{\circ} \mathrm{C},{ }^{25,29,31,36,37}$ attributed to the dimensionality effect associated with the lower coordination at the surface. ${ }^{25,29}$

The spatial dimension of polarization control in thin films of P(VDF-TrFE) has decreased substantially in the past decade. In 1992, Güthner and Dransfeld ${ }^{21}$ used the scanning force microscopy (SFM) to polarize P(VDF-TrFE) copolymer films. The smallest region they could polarize was about $1 \mu \mathrm{m}$. Similar results were obtained by poling and the imaging with an AFM. ${ }^{22,35,37-39}$ Kimura et al. ${ }^{40}$ obtained a size of $0.3 \mu \mathrm{m} \times 0.3 \mu \mathrm{m}$ polarized area. In 1998, Matsushige et $a l .{ }^{41}$ reported that the ferroelectric domains of P(VDF-TrFE) could be formed locally with dimensions of about $30 \mathrm{~nm}$ in diameter by AFM. More recently, Noda and co-workers ${ }^{11}$ also found that they could polarize a region about $65 \mathrm{~nm}$ in VDF oligomer films using an AFM. Lately $\mathrm{Qu}$ and co-workers ${ }^{30}$ achieved nanoscale polarization manipulation and spontaneous conductance switching in ultrathin P(VDFTrFE) films. Yet the effect of polarization control on the local structure needs to be addressed and investigated, as polarization manipulation becomes more and more localized to individual monomers.

In this paper, we present the detailed studies of surface structure and structure distortion associated with the polarization manipulation induced by STM. The surface of ultrathin and high quality crystalline $\mathrm{P}(\mathrm{VDF}-\mathrm{TrFE})$ films, fabricated on graphite substrates by the Langmuir-Blodgett (LB) technique, exhibits a quasihexagonal close packing structure with long-range polymer chain ordering, indicating a strong structural relationship between the polymer films and the substrate. Furthermore, a quasispiral twist on the intrachain structure was observed, possibly as a result of the alignment of polymer units (or monomers) at different canting angles. This twistlike structure may have a direct connection to the observed STM tip-induced local dipole reorientation and nanoscale conductance switching. ${ }^{30}$ 


\section{EXPERIMENTAL DETAILS}

$\mathrm{P}(\mathrm{VDF}-\mathrm{TrFE})$ films used in this study were crystalline with very large domains of oriented copolymer chains consisting of $70 \%-\mathrm{CH}_{2}-\mathrm{CF}_{2}-$ (vinylidene) and $30 \%$ $-\mathrm{CHF}-\mathrm{CF}_{2}-$ (trifluoroethylene) monomers. The highly crystalline and well ordered $\mathrm{P}(\mathrm{VDF}-\mathrm{TrFE})$ films were made by the horizontal LB monolayer transfer technique. ${ }^{18,23}$ At room temperature, the films were made by transferring two $\mathrm{P}(\mathrm{VDF}-\mathrm{TrFE})$ layers to freshly cleaved highly oriented pyrolytic graphite (HOPG) surface. Such films show the maximum spontaneous polarization $P \sim 0.1 \mathrm{C} \mathrm{m}^{-2}$ and large thermal hysteresis in thick films. ${ }^{26}$

After being transferred into the ultrahigh vacuum (UHV) chamber, the sample was cleaned by gently annealing at $\sim 420 \mathrm{~K}$ in an ultrahigh vacuum (UHV) chamber. The vacuum annealing at $\sim 420 \mathrm{~K}$ not only cleaned the surface of the sample, but also increased the crystallinity of P(VDFTrFE) films. ${ }^{29}$ The global ordering of similar films has been characterized using low-energy electron diffraction (LEED). ${ }^{26,29-31}$ STM measurements were done with the base pressure lower than $2 \times 10^{-10}$ Torr. A sharp W tip was used to obtain atomic resolution image, reorient the dipole moment(s) and measure $I-V$ curves. All the STM studies reported herein were done at room temperature by an Omicron variable-temperature STM.

\section{RESULTS AND DISCUSSION}

\section{A. Surface lattice structure}

The basic units (or monomers) of [P(VDF-TrFE)] chains, $-\mathrm{CH}_{2}-\mathrm{CF}_{2}-$ (vinylidene) and $-\mathrm{CHF}-\mathrm{CF}_{2}-$ (trifluoroethylene), have permanent dipole moments pointing from electronegative fluorine to the electropositive hydrogen where $-\mathrm{CH}_{2} /-\mathrm{CHF}$ - clusters act as the charge-positive side of the dipole while $-\mathrm{CF}_{2}-$ plays as the chargenegative side of the dipole. ${ }^{24-27,34,42}$ While the $\mathrm{P}(\mathrm{VDF}-\mathrm{TrFE})$ polymer chains in the bulk and in the thin films may adopt a number of different configurations, the ferroelectric and paraelectric phases are exemplified by two different monomer arrangement configurations below or above the bulk phase transition temperature $\left(T_{C}{ }^{B}\right)$, respectively. In the ferroelectric $\beta$ phase, below $T_{C}{ }^{B}, \mathrm{P}(\mathrm{VDF}-\mathrm{TrFE})$ chains adopt an all-trans (TTTT) configuration [Fig. 1(b)] where each monomer is aligned roughly perpendicular to the polymer chain axis. ${ }^{26,27,33,34,42,43}$ In the paraelectric $\alpha$ phase, above $T_{C}{ }^{B}$, the $\mathrm{P}(\mathrm{VDF}-\mathrm{TrFE})$ chains have a random alternating trans-gauche structure $(\mathrm{TG})_{0.5}(\mathrm{~T} \bar{G})_{0.5}$, which has no net dipole moment. ${ }^{26,32-34}$

A typical atomic resolution image of the morphology of thin $\mathrm{P}(\mathrm{VDF}-\mathrm{TrFE})$ films on graphite at room temperature is shown in Fig. 1(a), measured by STM at $-0.36 \mathrm{~V}$ tip bias. Every bright protrusion represents either $-\mathrm{CH}_{2}-1-\mathrm{CHF}-$ or $-\mathrm{CF}_{2}-$ cluster in a monomer, ${ }^{18,24,30}$ though we are not able to distinguish $-\mathrm{CH}_{2}-$ from $-\mathrm{CHF}-$ in the images. In Fig. 1(a), it can be seen that these monomers arranged themselves in parallel rows, forming a quasihexagonal close packing structure. By calibrating the dimensions in this image with the image of a
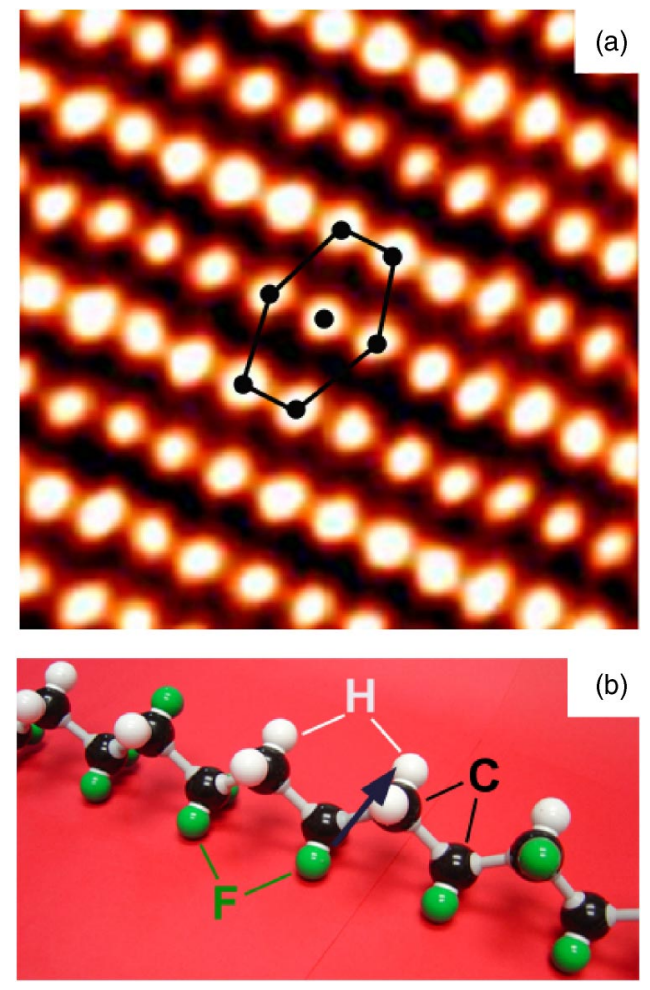

FIG. 1. (Color-online) (a) An atomically resolved STM image of the surface of $\mathrm{P}(\mathrm{VDF}-\mathrm{TrFE}$ 70:30) film on graphite at room temperature and (b) Ball structural model for a $\mathrm{P}(\mathrm{VDF}-\mathrm{TrFE})$ chain in the all-trans configuration. Every bright spot represents a $-\mathrm{CH}_{2}-\mathrm{CF}_{2}-$ or $-\mathrm{CHF}-\mathrm{CF}_{2}-$ monomer. The tip-bias voltage $V_{\text {bias }}$ and the tunneling current $I$ were $-0.36 \mathrm{~V}$ and $0.38 \mathrm{nA}$, respectively. The image size is about $2.6 \mathrm{~nm} \times 2.6 \mathrm{~nm}$. The arrow shows the direction of monomer dipole moment. The surface unit cell is also indicated.

freshly cleaved graphite surface acquired under the same experimental conditions, we obtained that the intrachain monomer-monomer spacing $a$, i.e., the lattice constant along chain direction, was $2.4 \pm 0.1 \AA$. This is in good agreement with the previous result $(2.5 \pm 0.1 \AA$ ) (Ref. 29) measured by low-energy electron diffraction (LEED) and photoemission, but is potentially a little smaller than the $2.55 \AA$ observed in diffraction studies of thick films or the bulk material. ${ }^{32,42}$ However, the average interchain spacing $b$, the lattice constant perpendicular to chains, was only about $3.3 \pm 0.1 \AA$. This is much smaller than the bulk close packing spacing between chains of $4.56 \AA .^{29,32}$ Notice that in Fig. 1(a), the position of the $-\mathrm{CH}_{2}-\mathrm{CF}_{2}-$ monomers in neighboring $\mathrm{P}(\mathrm{VDF}-\mathrm{TrFE})$ chains slips by one-quarter of the intrinsic dimer super periodicity or $\left(\frac{1}{2}\right)$ of intrachain spacing along the chain axis. Both the LEED and x-ray scattering data ${ }^{26,29}$ from the high order diffraction beams, provide evidence of a superperiodicity both in the plane and along the surface normal, with the in-plane superperiodicity consistent with the one-half $\left(\frac{1}{2}\right)$ slip in the intrachain spacing along the chain axis observed in the STM images. The subtle details and fine structure of intra polymer chains structure and monomer orientation, as discussed below, are largely absent, nonetheless, some inferences can be made concerning the P(VDF-TrFE) 


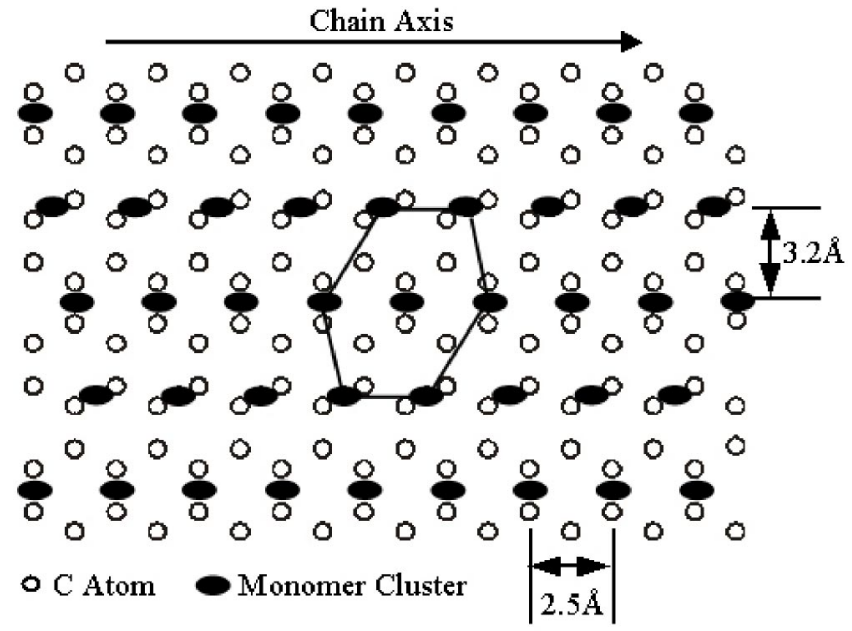

FIG. 2. A proposed structure of P(VDF-TrFE) on graphite. Hollow circles represent substrate carbon atoms while filled circles represent copolymer monomers.

chain to substrate interaction based on these images obtained at modest $(>300 \mathrm{meV})$ bias voltages.

When atoms or molecules adsorb on the substrate, the adsorption sites of adsorbates are determined by both the adsorbate-adsorbate and adsorbate-substrate interactions. If we only consider the graphite substrate surface, there are three different high symmetry adsorption sites. One such site is the hollow site located above the middle of the sixfold hexagonal graphite ring. Another one high symmetry site is the top site which is positioned on the top of underlying carbon atom of the surface layer. The third such high symmetry site is the bridge site located on the middle of the shortest $\mathrm{C}-\mathrm{C}$ bond. While it is unlikely that all monomers of the $\mathrm{P}(\mathrm{VDF}-\mathrm{TrFE})$ chains occupy the same surface adsorption sites on the graphite surface, due to the large lattice mismatches for the interchain spacing $(b)$, the interaction between the P(VDF-TrFE) chains and the surface of graphite is likely to be strong. Interaction induced registry with the substrate can occur because the intrachain lattice spacing $(a)$ is close in value to the lattice constant of graphite surface along [1 100$]$ direction. While there is a large lattice mismatch in the interchain spacing $(b)$ with the substrate, thus substrate induced strain in the $\mathrm{P}(\mathrm{VDF}-\mathrm{TrFE})$ chain adlayer remains.

Considering the symmetry of the structure and the measured values of $a$ and $b$ by STM, we propose a possible structure schematically shown in Fig. 2. In this schematic adlayer structure, the $\mathrm{P}(\mathrm{VDF}-\mathrm{TrFE})$ chain $-\mathrm{CF}_{2}-$ units occupy only the graphite bridges sites, while the monomers on the neighboring $\mathrm{P}(\mathrm{VDF}-\mathrm{TrFE})$ chains occupy at different bridge sites. Meanwhile, this proposed structure (Fig. 2) for $\mathrm{P}(\mathrm{VDF}-\mathrm{TrFE})$ graphite preserves the quasihexagonal symmetry with the intrachain spacing and interchain spacing are 2.5 and $3.2 \AA$, respectively, consistent with results obtained from STM images. Furthermore, it is obvious that there is a shift of $\left(\frac{1}{2}\right)$ in intrachain spacing along the chain axis for the neighbor monomers, as seen from the STM image and the proposed structure in Fig. 2. As noted above, Choi and co-
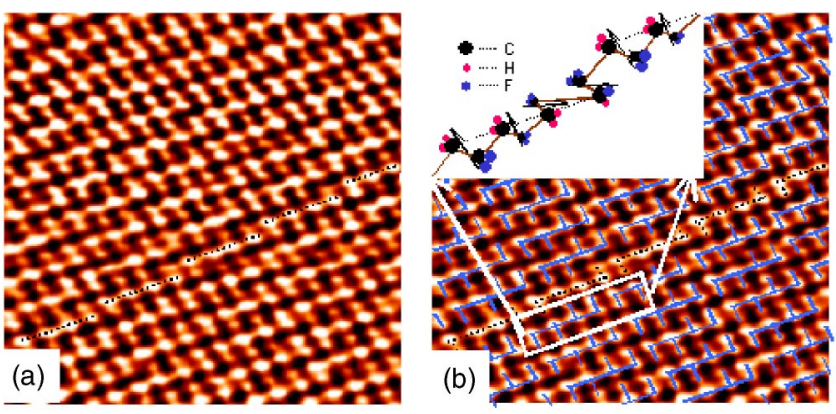

FIG. 3. (Color online) (a) STM image of P(VDF-TrFE) on graphite at room temperature obtained with $V_{\text {bias }}=-0.10 \mathrm{~V}$ and $I$ $=0.21 \mathrm{nA}$. The image size is $4.4 \mathrm{~nm} \times 4.4 \mathrm{~nm}$. A fine intra-chain structure is shown in the image. The dashed black lines only guide the eye. (b) The same picture as Fig. 3(a) but with the grid showing the superstructure of the surface. The short solid blue or solid black line indicates two protrusions that belong to one monomer. The long dashed black line indicates the domain boundary. The inset shows the schematic intra-chain structure with four possible directions of monomers in the $(4 \times 1)$ superlattice. The black arrows indicate directions of dipole moment. The dashed black lines only guide the eye.

workers reported similar monomer displacements from one chain to the next, ${ }^{29,31}$ although a different interpretation from what is proposed here was put forward because there were insufficient details in the real space images.

The strong strain in the film due to the compacted structure with much smaller interchain spacing is expected to be relieved with increasing film thickness through the introduction of defects and disorder. ${ }^{43-52}$ Indeed, disorder in the interchain spacing of the $\mathrm{P}(\mathrm{VDF}-\mathrm{TrFE})$ chains has been observed for LB deposited films of $\mathrm{P}(\mathrm{VDF}-\mathrm{TrFE})$ on both graphite and $\mathrm{Si}(111),{ }^{26,29,31}$ where the range of the interchain space has been observed to vary from 3.5 to $4.3 \AA$. This prior evidence $26,29,31$ of disorder of the interchain spacing occurred while ordering along the chain is preserved, is consistent with our suggested model of the adlayer structure.

\section{B. Intrachain superlattice formation}

The competition between extreme strain and strain relief can evolve into quasisuperlattice structures in some surface regions. ${ }^{45,46}$ We have indeed observed a delicate superlattice structure with quasispiral dipole twist about the chain direction in the thin P(VDF-TrFE) films. This superlattice structure was observed in the STM images of the surface of $\mathrm{P}(\mathrm{VDF}-\mathrm{TrFE})$ films, at room temperature, when we brought STM tip close to the surface by employing small bias voltages. The STM image shown in Fig. 3(a) was obtained with the tip bias $V_{\text {bias }}=-0.10 \mathrm{~V}$ and the tunneling current $I$ $=0.21 \mathrm{nA}$, respectively, so that the tip-induced field near the $\mathrm{P}(\mathrm{VDF}-\mathrm{TrFE})$ film surface was relatively weak. Such a superstructure was observed only with low tip bias, indicating that the strong tip-induced fields, occurring with large bias voltages, may destroy the superstructure.

These low bias STM images are drastically different from the STM images obtained with higher bias, as shown in Fig. 1. First, a clear intrachain structure is evident with protru- 
sions in uneven brightness. Second, the bright protrusions appear simultaneously accompanied by the weaker protrusions with a modulation of four times the periodicity of monomers along a copolymer chain (see Fig. 3). Thirdly, along the chain axis, almost every four bright spots are shifted a little distance perpendicular to the chain direction in the image [as shown in Fig. 3(b), with a grid to guide the eye]. The blue lines in Fig. $3(\mathrm{~b})$ indicate this $(4 \times 1)$ superstructure.

As mentioned above, every protrusion shown in Fig. 1(a) represents either $-\mathrm{CH}_{2}-/-\mathrm{CHF}-$ or the $-\mathrm{CF}_{2}-$ unit of a monomer without the detailed structure revealed in Fig. 3. The differences between Figs. 1 and 3, we believe, results from the differences in the image conditions. A sufficiently large tip field (between the STM tip and the film) will perturb the orientation of surface dipole moments thus change the apparent surface structure. When the static external electric field is strong enough, the dipoles formed from monomers tend to align themselves normal to the surface. Only one side of the monomer, either the $-\mathrm{CH}_{2}-/-\mathrm{CHF}-$ or $-\mathrm{CF}_{2}-$ cluster, can be imaged as shown in Fig. 1(a). Such images may also reflect the perturbations in the dipole orientation by the moving STM tip as the image is taken.

With weaker STM tip fields, ideally more representative of the surface electron density, hopefully the STM images are more representative of a largely unperturbed surface structure. Under very weak STM tip fields (low enough tip bias), both $-\mathrm{CH}_{2}-1-\mathrm{CHF}-$ and $-\mathrm{CF}_{2}-$ clusters, i.e., the both sides of an individual monomer may be observed simultaneously, though depending on whether or not local net dipole moment in the monomer is canted away from the surface normal. We believe that Fig. 3 indeed shows the image consisted of both $-\mathrm{CH}_{2}-\mathrm{I}-\mathrm{CHF}-$ and $-\mathrm{CF}_{2}-$ clusters, reflecting the intrinsic structure of the surface copolymer layer. However, the image (Fig. 3) did not resolve each hydrogen or fluorine atom. Otherwise we should see the differences of randomly distributed $-\mathrm{CHF}$ - clusters from $-\mathrm{CH}_{2}-$ clusters.

Based on the measurement parameters, especially the negative bias polarity of the STM tip, we conclude that the brighter protrusions in Fig. 3 represent the $-\mathrm{CH}_{2}-/-\mathrm{CHF}$ - cluster (the charge-positive part of monomer) while the weaker protrusions represent the $-\mathrm{CF}_{2}-$ clusters (the charge-negative part of the monomer). The simultaneous observation of both positive and negative parts of the dipole indicates that the dipole moment of the $-\mathrm{CH}_{2}-\mathrm{CF}_{2}-$ monomers lie at a canting angle with respect to the surface normal in the low bias STM images. The observed variations in the apparent distances between the protrusion of $-\mathrm{CH}_{2}-/-\mathrm{CHF}-$ clusters and the protrusion of $-\mathrm{CF}_{2}-$ clusters in the STM image may reflect various canting angles possibly caused by the rotation of the $-\mathrm{CH}_{2}-\mathrm{CF}_{2}-$ monomers about the chain axis [see the inset of Fig. 3(b)]. The variation in brightness ratio between $-\mathrm{CH}_{2}-1-\mathrm{CHF}-$ and $-\mathrm{CF}_{2}-$ protrusion reveals the changes of the relative height between $-\mathrm{CH}_{2}-/-\mathrm{CHF}-$ and $-\mathrm{CF}_{2}-$ cluster for different monomers, though variations in charge density cannot be completely excluded in spite of the good dielectric properties of this polymer. ${ }^{53}$

The observed $4 \times 1$ superlattice reflects a periodic canting of $-\mathrm{CH}_{2}-\mathrm{CF}_{2}-$ monomer dipoles resulting from rota- tions about the chain axis, thus forming a "twisted" $\mathrm{C}-\mathrm{C}$ bond structure. One such scheme is schematically shown in the inset of Fig. 3(b), though limited only by steric hindrance and dipole coupling. ${ }^{43}$ Such a "twisting" structure by rotation of the dipole moments back and forth about the chain axis will generally preserve the conventional bond angle as well as maintain the average expected bond length. A clear mechanism behind this back and forth twisting structure has yet to be resolved. Both the static effects due to a surface lattice relaxation as well as the close proximity in temperature to the surface ferroelectric phase transition $\left(T_{C}^{S}\right)^{25,29,31,35-37}$ may play a role. LB thin films of P(VDFTrFE) in the $\beta$ phase is a ferroelectric phase with a dipole alignment along the surface normal. ${ }^{43,54}$ However, it is not necessary that, in the room temperature thin films, all of dipoles have the same direction under absent or low external electronic fields, especially at the temperature near $T_{C} S$ $\left(\sim 20^{\circ} \mathrm{C}\right) .{ }^{24-26,29}$ This $4 \times 1$ structure is certainly consistent with the doubling of the periodicity along the polymer chain observed above $T_{C}{ }^{S} \cdot 31$

The influence of the conducting substrate will tend to orient the dipole direction along the surface normal, ${ }^{43}$ although the recent theoretical studies by Duan et al. ${ }^{43}$ and experimental studies ${ }^{55}$ indicate insulating substrates leads to the tendency that places the dipole orientation away from the surface normal. The strong dipole-dipole coupling, the adlayersubstrate interactions, as well as the strain effects, may result in a compromised canted structure at the surface. This may explain the difference of surface phase transition temperature from that in the bulk. On the other hand, strong temperature dependence of the dipole stiffness in $\mathrm{P}(\mathrm{VDF}-\mathrm{TrFE})$ chains $^{53}$ and other quasi-one-dimensional dipole chains ${ }^{56}$ have been observed and are expected to play a role near $T_{C}{ }_{C}$. If such is the case, then the dipole stiffness should also depends upon the extrinsic field conditions.

\section{STM-induced structural distortions}

Local external electronic fields can affect the orientation of the monomer dipole moments of $\mathrm{P}(\mathrm{VDF}-\mathrm{TrFE})$ films, ${ }^{11,22,30,35-41}$ thus resulting in local polarization and local switching (dipole reversal). ${ }^{30}$ In previous experiments, the polarization alignment and conductance switching were achieved by changing the value and the polarity of the tipbias voltage. ${ }^{30}$ The reversal of surface polarization induced by STM tip field is clearly evident in an apparent local lattice distortion in the topographic images by STM. Such a distortion, as we discuss below, is directly related to the rotational freedom of dipole moments about the polymer chain axis.

The results of the lattice distortion induced by STM are shown in Fig. 4. An apparent lattice shift occurs simultaneously when flipping the polarity of STM tip bias and when scanning direction is largely perpendicular to the polymer chain axis. The image of Fig. 4(a) was taken in the following process. During scanning from the bottom of the image, the tip-bias $V_{\text {bias }}$ was first set at $+0.57 \mathrm{~V}$. After the $1 / 3$ of the surface area was scanned, the tip-bias $V_{\text {bias }}$ was flipped to $-0.57 \mathrm{~V}$ and continued to scan another $1 / 3$ of the surface area. At last the tip-bias $V_{\text {bias }}$ was back to $+0.57 \mathrm{~V}$ to scan 

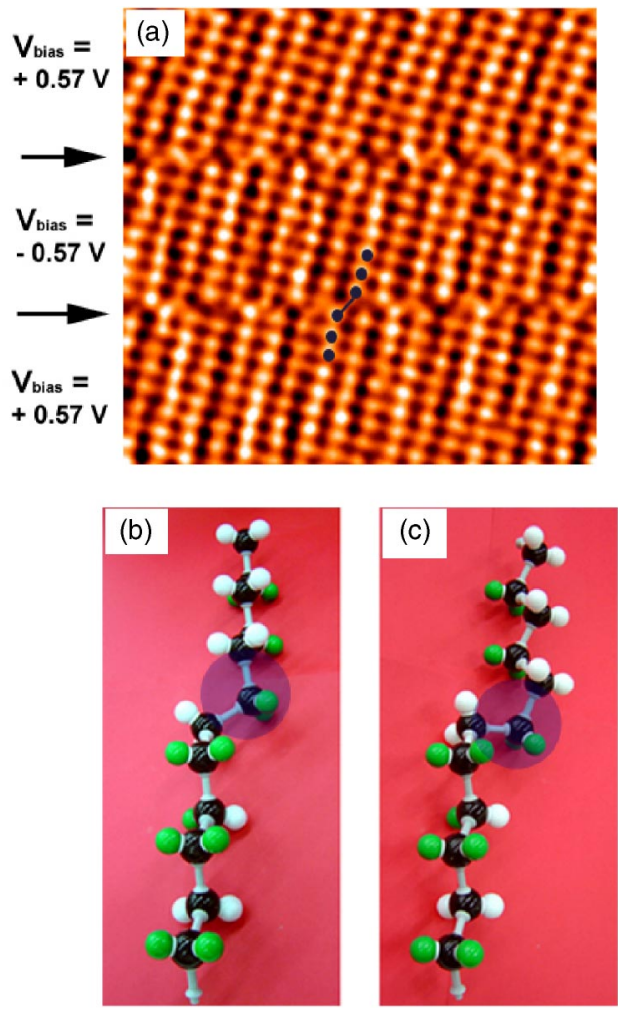

FIG. 4. (Color online) (a) The STM image on a P(VDF-TrFE) copolymer film at room temperature obtained by flipping the polarity of the tip bias voltage at different values. Image size is $4.4 \mathrm{~nm}$ $\times 4.4 \mathrm{~nm}$. The polarity of the tip bias voltage was flipped from $0.57 \mathrm{~V}$ to $-0.57 \mathrm{~V}$ and then back to $0.57 \mathrm{~V}$ during measurement. The tunneling current was $0.6 \mathrm{nA}$. The image of $-\mathrm{CH}_{2} / \mathrm{CHF}-$ or $-\mathrm{CF}_{2}$ - clusters are indicated. (b) The "flip-reversal" model and (c) "cant-reversal" model. The two C-C bonds involved in the "twisting" distortion induced by STM are indicated in the circular shadows.

the rest area. When flipping tip-bias voltage, a shift of the bright protrusions, by about half of interchain spacing (b), is evident in the image. Indeed, the lattice shift was observed only with tip-bias voltages in the range from 0.1 to $1.0 \mathrm{~V}$ and when the tip-sample gap remained roughly constant by keeping the ration of $V_{\text {bias }} / I$ constant. The sharp induced local polarization boundary [indicated by arrows in Fig. 4(a)] was observed as a consequence of flipping the local field through reversing the bias applied to the STM tip. We found the image conditions, with $\left|V_{\text {bias }}\right| \sim 0.57 \mathrm{~V}$, were the best for obtaining such an apparent shift. To ensure that the observed phenomena on the surface of P(VDF-TrFE) films is not an artificial effect, we have also confirmed that there is no apparent lattice shift by flipping tip bias exhibited the images of clean graphite surface with the same image conditions.

We have also applied different scanning procedures with various $V_{\text {bias }}$ to scan the surface. We found that the lattice shift was observed only in the tip-bias voltage range from 0.1 to $1.0 \mathrm{~V}$ when the tip-sample gap remained roughly constant by keeping the ration of $V_{\text {bias }} / I$ constant. We also found no clear shift of relative positions was observed when the scan direction was parallel to the chain axis, regardless of the magnitude of the change in the tip bias $V_{\text {bias. }}$.
The net polarization towards the vacuum with no external electric field is largely maintained ${ }^{25,29,31}$ or enhanced by a negative tip bias which will compel the local dipole moment underneath the tip to polarize along the surface normal with the charge-positive $-\mathrm{CH}_{2}-/-\mathrm{CHF}-$ cluster towards the vacuum. Using similar reasoning, the surface dipole moments will orient to the opposite direction with the $\mathrm{CH}_{2}-/-\mathrm{CHF}$ - cluster towards the substrate if the tip bias is positive. We suggest that this observed lattice shift is directly linked to a field-induced local structure distortion: the tip field results in a local dipole moment rotation accompanied by a spontaneous change of local structure, at least in the top layer of the film.

There are most likely two contributions to the observed structural distortion induced by a moderate STM tip field. The ball-models for these two possibilities are shown in the Figs. 4(b) and 4(c), one is referred to as the "flip-reversal" model and the other as the "cant-reversal" model. The - $\mathrm{C}-\mathrm{C}$ - bond angle natural to all alkane chains will result in a displacement as the dipole is reversed, while changes in the canting or tilting of the dipoles will also affect the apparent local structure in the STM image. To reverse the dipole with the minimum number of $-\mathrm{C}-\mathrm{C}-$ bonds, while keeping the $\mathrm{C}-\mathrm{C}$ bond angle intact and the polymer chains from buckling out the surface plane, requires a lattice distortion (or "twist") involving at least two $-\mathrm{C}-\mathrm{C}-$ bonds, as indicated in the circle shadows in Figs. 4(b) and 4(c). Such a dipole "reversal" causes a translational displacement largely perpendicular to the chain directions (i.e., in the surface plane) as schematically shown in Figs. 4(b) and 4(c).

A dipole reversal mechanism that exploits only one - $\mathrm{C}-\mathrm{C}-$ bond "twist" would result in no lateral displacement but a displacement along the surface normal, causing a buckling of the polymer chains during reversing the polarization. Energetically, this requires a strong tip field with a large $V_{\text {bias. }}$. This may explain why the apparent lattice shift was not observed when $V_{\text {bias }} \geqslant 1.0 \mathrm{~V}$ which cannot be understood with these models shown in Figs. 4(b) and 4(c).

As shown in Figs. 4(b) and 4(c), both models provide similar lateral displacements perpendicular to the polymer chains as observed in the STM images. The "flip-reversal" model presents simply the flipping of two carbon positions in a single monomer, that is the $\mathrm{CF}_{2}$ switches to the top while $\mathrm{CH}_{2} / \mathrm{CHF}$ to the bottom in the polarized monomer, without any canting of dipole moments [see Fig. 4(b)]. Such a situation might be caused by larger extrinsic tip fields since the dipole moments are completely reversed by reversing the tip bias polarity. As has been indicated by the surface structural studies discussed above, dipole reorientation or canting is favored by rotations largely perpendicular to the chain direction, thus favoring a lattice shift only when the scan direction is also largely perpendicular to the chain. This suggests the other possible mechanism, the "cant-reversal" model, in which the dipole moments are canted from the surface normal before and after tip-induced reversal. This mechanism may be energetically favorable since the dipole moments are partially reversed by STM. It can be expected that the canting angle depends on the dipole stiffness and the tip bias $V_{\text {bias. }}$. Although the "cant-reversal" model should have a smaller "gap" at the induced domain boundary (marked by 
the arrows in Fig. 4) than the "flip-reversal" model, we are unable to distinguish them based on our experimental data.

No clear apparent shift was observed when scanning in the direction parallel to the chain axis does not mean that there is no polarization reversal by flipping tip bias. When scanning across the polymer chains, all the chains are involved in the spontaneous process of flipping. In contrast, when scanning along the chain direction, only one single chain may be involved during flipping the tip bias. This makes the shift hard to be observed more steric hindering to rotation is anticipated with this scan direction.

As mentioned above, observed obvious lattice shift occurred only when $\left|V_{\text {bias }}\right| \geqslant 0.1 \mathrm{~V}$. Based upon the energetic point of view, a certain switching energy is needed to overcome the energy barrier for the dipole rotation, thus a minimum tip field is required for the polarization. This explains why no apparent shift was observed when $\left|V_{\text {bias }}\right| \leqslant 0.1 \mathrm{~V}$ which should correspond to the required minimum bias voltage. Clearly, this also explain why a superstructure can be observed only with $\left|V_{\text {bias }}\right| \leqslant 0.1$ as described in previous section. Estimating 3-5 $\AA$ of the tip-sample gap, the minimum electronic field induced dipole rotation is about $0.2-0.4 \mathrm{G} \mathrm{V} \mathrm{m}^{-1}$ for the lower limit of the tip-bias voltage $0.1 \mathrm{~V}$. This value is very close to the coercive field of $0.5 \mathrm{G} \mathrm{V} \mathrm{m}^{-1}$ for a $2 \mathrm{ML}$ film obtained by the pyroelectric technique. $^{24,26}$

Due to the interaction with the substrate, it is believed that the monomers in the bottom layer have the fluorine oriented towards while the hydrogen away from the substrate. ${ }^{24-26,40}$ By scanning with negative tip bias, the imaged monomers in the top layer are spontaneously polarized roughly parallel to these in the bottom layer. On the other hand, the monomer dipole moments between the two layers are more or less in opposite direction when imaging with positive tip bias, in our proposed model of local dipole reversal. Note that there is anisotropy of the intrinsic potential barrier for flipping dipole parallel to and antiparallel to the bottom layer. Ignoring dynamics and just considering the activation energies, it is expected that a longer time may be needed to switch dipole moments in the top layer to be antiparallel to those on the bottom layer. However, domain boundaries [indicated by the arrows in Fig. 4(a)] for the two different flipping processes are very sharp and almost identical, when reversing the polarity of the applied tip-bias voltage in the range of 0.1 to $1.0 \mathrm{~V}$. It can be inferred that at least in these thin $\mathrm{P}(\mathrm{VDF}-\mathrm{TrFE})$ films the induced dipole rotation is reasonably fast, at least faster than the time of the STM measurement. The fast switching is another reason that we observe the polarity-dependent lattice shift.

\section{CONCLUSION}

We have studied the thin crystalline $\mathrm{P}(\mathrm{VDF}-\mathrm{TrFE})$ copolymer films on graphite by STM. We observed the wellordered and compact structure of the ultrathin films that indicated the strain effect of the substrate. We observed a quasispiral twist structure of the copolymer chains, reflecting the relaxation of the lattice strain in the quasiferroelectric phase, and dipole canting away from the surface normal is implicated in the surface structure near room temperature as well as in the dipole reversal process. We demonstrate dipole orientation manipulation on the nanometer size scale. The behavior of the surface structure, polarization-induced lattice shift, as well as conductance switching can be ascribed to the local dipole moment reversal due to the rotational degree of freedom in the plane perpendicular to the copolymer chain axis. The character of the sharp domain boundary suggests that $\mathrm{P}(\mathrm{VDF}-\mathrm{TrFE})$ may be a candidate material for the high capacity data storage, if such dipole domains on the nanometer scale can be controlled and remain stable. The latter point requires further investigation.

\section{ACKNOWLEDGMENTS}

This work was supported by the Petroleum Research Fund (Grant No. 36777-G5) administrated by the ACS, the NSF through Grant No. DMR-0346826, CHE-0415421 and the NSF "QSPINS" MRSEC (Grant No. DMR-0213808). The authors would like to acknowledge a number of helpful conversations with V. Fridkin, and some technical assistance from Luis Rosa.
*Current address: Department of Electrical and Computer Engineering, the University of Florida

$\dagger$ Author to whom correspondence should be addressed. Electronic address: zhangj@fiu.edu

${ }^{1}$ G. Binnig and H. Rohrer, Rev. Mod. Phys. 59, 615 (1987).

${ }^{2}$ G. Binnig, C. F. Quate, and C. Gerber, Phys. Rev. Lett. 56, 930 (1986); D. Sarid, Scanning Force Microscopy (Oxford University Press, New York, 1994).

${ }^{3}$ D. M. Eigler and E. Schweizer, Nature (London) 344, 524 (1990).

${ }^{4}$ J. A. Stroscio and D. M. Eigler, Science 254, 1319 (1991).

${ }^{5}$ L. Bartels, G. Meyer, and K. H. Rieder, Phys. Rev. Lett. 79, 697 (1997).

${ }^{6}$ P. Beton, A. Dunn, and P. Moriarty, Surf. Sci. 361-362, 878
(1996).

${ }^{7}$ Ch. Loppacher, M. Guggisberg, O. Pfeiffer, E. Meyer, M. Bammerlin, R. Lüthi, R. Schlittler, J. K. Gimzewski, H. Tang, and C. Joachim, Phys. Rev. Lett. 90, 066107 (2003).

${ }^{8}$ H. Yanagi, K. Ikuta, H. Mukai, and T. Shibutani, Nano Lett. 2, 951 (2002).

${ }^{9}$ R. Lin, K.-F. Braun, H. Tang, U. J. Quaade, F. C. Krebs, G. Meyer, C. Joachim, K.-H. Rieder, and K. Stokbro, Surf. Sci. 477, 198 (2001).

${ }^{10}$ F. Moresco, G. Meyer, K.-H. Rieder, H. Tang, A. Gourdon, and C. Joachim, Phys. Rev. Lett. 86, 672 (2001).

${ }^{11}$ K. Noda, K. Ishida, A. Kubono, T. Horiuchi, H. Yamada, and K. Matsushige, Jpn. J. Appl. Phys., Part 1 40, 4361 (2001).

${ }^{12}$ H. J. Gao, K. Sohlberg, Z. Q. Xue, H. Y. Chen, S. M. Hou, L. P. 
Ma, X. W. Fang, S. J. Pang, and S. J. Pennycook, Phys. Rev. Lett. 84, 1780 (2000).

${ }^{13}$ R. S. Potember, Molecular Electronic Devices, edited by F. L. Carter (Marcel Decker, New York, 1992), p. 73.

${ }^{14}$ F. Ebisawa, T. Kurosawa, and S. Nara, J. Appl. Phys. 54, 3255 (1983).

${ }^{15}$ H. J. Mamin, S. Chiang, H. Birk, P. H. Guethner, and D. Rugar, J. Vac. Sci. Technol. B 9, 1398 (1991).

${ }^{16}$ H. Kawai, Jpn. J. Appl. Phys. 8, 975 (1969).

${ }^{17}$ A. Bune, S. Ducharme, V. Fridkin, L. Blinov, S. Palto, N. Petukhova, and S. Yudin, Appl. Phys. Lett. 67, 3975 (1995).

${ }^{18}$ S. Palto, L. Blinov, E. Dubovik, V. Fridkin, N. Petukhova, A. Sorokin, K. Verkhovskaya, S. Yudin, and A. Zlatkin, Europhys. Lett. 34, 465 (1996).

${ }^{19}$ H. Ohigashi, S. Akama, and K. Koga, Jpn. J. Appl. Phys., Part 1 27, 2144 (1988).

${ }^{20}$ K. Kimura and H. Ohigashi, Jpn. J. Appl. Phys., Part 1 25, 383 (1986).

${ }^{21}$ P. Güthner, and K. Dransfeld, Appl. Phys. Lett. 61, 1137 (1992).

${ }^{22}$ T. Fukuma, K. Kobayashi, T. Horiuchi, H. Yamada, and K. Matsushige, Jpn. J. Appl. Phys., Part 1 39, 3830 (2000).

${ }^{23}$ S. Palto, L. Blinov, A. Bune, E. Dubovik, V. Fridkin, N. Petukhova, K. Verkhovskaya, and S. Yudin, Ferroelectr., Lett. Sect. 19, 65 (1995).

${ }^{24}$ A.V. Bune, V.M. Fridkin, S. Ducharme, L. M. Blinov, S. P. Palto, A. Sorokin, S.G. Yudi, and A. Zlatkin, Nature (London) 391, 874 (1998).

${ }^{25}$ J. Choi, P.A. Dowben, S. Pebley, A.V. Bune, S. Ducharme, V. M. Fridkin, S. P. Palto, and N. Petukhova, Phys. Rev. Lett. 80, 1328 (1998).

${ }^{26}$ L. M. Blinov, V. M. Fridkin, S. P. Palto, A. V. Bune, P. A. Dowben, and S. Ducharme, Phys. Usp. 43, 243 (2000).

${ }^{27}$ T. Furukawa, Phase Transitions 18, 143 (1989).

${ }^{28}$ S. Ducharme, A. V. Bune, L. M. Blinov, V. M. Fridkin, S. P. Palto, A. V. Sorokin, and S. G. Yudin, Phys. Rev. B 57, 25 (1998).

${ }^{29}$ J. Choi, C. N. Borca, P. A. Dowben, A. Bune, M. Poulsen, S. Pebley, S. Adenwalla, S. Ducharme, L. Robertson, V. M. Fridkin, S. P. Palto, N. N. Petukhova, and S. G. Yudin, Phys. Rev. B 61, 5760 (2000).

${ }^{30}$ Hongwei Qu, T. Garcia, W. Yao, Jiandi Zhang, S. Ducharme, P. A. Dowben, A. V. Sorokin, and V. M. Fridkin, Appl. Phys. Lett. 82, 4322 (2003).

${ }^{31}$ J. Choi, P. A. Dowben, S. Ducharme, V. M. Fridkin, S. P. Palto, N. Petukhova, and S. G. Yudin, Phys. Lett. A 249, 505 (1998).

${ }^{32}$ E. Bellet-Amalric and J. F. Legrand, Eur. Phys. J. B 3, 225 (1998).

${ }^{33}$ G. T. Davis, in The Applications of Ferroelectric Polymers, edited by T. T. Wang, J. M. Herbert, and A. M. Glass (Bell \& Bain, Glasgow, 1988), Chap. 9, p. 37.

${ }^{34}$ S. Ducharme, S. P. Palto, V. M. Fridkin, and L. M. Blinov, in Handbook of Surfaces and Interfaces of Materials, Vol. 3, Chap. 11.

${ }^{35}$ V. M. Fridkin, S. Ducharme, A. V. Bune, S. P. Palto, and L. M. Blinov, Ferroelectrics 236, 1 (2000).

${ }^{36}$ O. A. Aktsipetrov, L. M. Blinov, V. M. Fridkin, T. V. Misuryaev, T. V. Murzina, S. P. Palto, and S. G. Yudin, Surf. Sci. 454, 1016 (2000).

${ }^{37}$ O. A. Aktsipetrov, T. V. Misuryaev, T. V. Murzin, L. M. Blinov, V. M. Fridkin, and S. P. Palto, Opt. Lett. 25, 411 (2000).

${ }^{38}$ X. Q. Chen, H. Yamada, Y. Terai, T. Horiuchi, K. Matsysguge, and P. S. Weiss, Thin Solid Films 353, 259 (1999).

${ }^{39}$ K. Noda, K. Ishida, A. Kubono, T. Horiuchi, H. Yamada, and K. Matsushige, Jpn. J. Appl. Phys., Part 1 39, 6358 (2000).

${ }^{40}$ K. Kimura, K. Kobayashi, H. Yamada, T. Horiuchi, K. Ishida, and K. Matsushige, Appl. Phys. Lett. 82, 4050 (2003).

${ }^{41}$ K. Matsushige, H. Yamada, H. Tanaka, T. Horiuchi, and X. Q. Chen, Nanotechnology 9, 208 (1998).

${ }^{42}$ A. J. Lovinger, Science 220, 1115 (1983).

${ }^{43}$ C.-G. Duan, W. N. Mei, Wei-Guo Yin, J. Liu, J. R. Hardy, S. Ducharme, and P. A. Dowben, Phys. Rev. B (to be published).

${ }^{44}$ R. W. Vook, Int. Met. Rev. 27, 209 (1982).

${ }^{45}$ E. Bauer, Appl. Surf. Sci. 11-12, 479 (1982).

${ }^{46}$ J. H. van der Merwe, Thin Solid Films 74, 129 (1980).

${ }^{47}$ F. C. Frank and J. H. van der Merwe, Proc. R. Soc. London, Ser. A 198, 205 (1949).

${ }^{48}$ L. Di Gaspare, A. Notargiacomo, F. Evangelisti, E. Palange, S. Pascarelli, and J. Susini, Solid State Commun. 122, 359 (2002).

${ }^{49}$ T. C. Rojas, S. I. Molina, A. Sacedon, F. Valtuena, E. Calleja, and R. Garcia, Thin Solid Films 317, 270 (1998).

${ }^{50}$ J. Groenen, G. Landa, R. Carles, P. S. Pizani, and M. Gendry, J. Appl. Phys. 82, 803 (1997).

${ }^{51}$ M. Hornvonhoegen, M. Pook, A. Alfalou, B. H. Muller, and M. Henzler, Surf. Sci. 284, 53 (1993).

${ }^{52}$ G. Meyer, B. Voigtlander, and N. M. Amer, Surf. Sci. 274, L541 (1992).

${ }^{53}$ C. N. Borca, S. Adenwalla, J. Choi, P. T. Sprunger, S. Ducharme, L. Robertson, S. P. Palto, J. Liu, M. Poulsen, V. M. Fridkin, H. You, and P. A. Dowben, Phys. Rev. Lett. 83, 4562 (1999).

${ }^{54}$ J. Choi, S.-J. Tang, P. T. Sprunger, P. A. Dowben, V. M. Fridkin, A. V. Sorokin, S. P. Palto, N. Petukhova, and S. G. Yudin, J. Phys.: Condens. Matter 12, 4735 (2000).

${ }^{55}$ K. Noda, K. Ishida, T. Horiuchi K. Matsushige, and A. Kuboono, J. Appl. Phys. 86, 3688 (1999).

${ }^{56}$ Ya. B. Losovyj, I. N. Yakovkin, H.-K. Jeong, D. Wisbey, and P. A. Dowben, J. Phys.: Condens Matter. (to be published). 